\title{
Stratifying the Singularity Loci of a Class of Parallel Manipulators
}

\author{
Carme Torras, Federico Thomas, and Maria Alberich-Carramiñana
}

\begin{abstract}
Some in-parallel robots, such as the 3-2-1 and the 3/2 manipulators, have attracted attention because their forward kinematics can be solved by three consecutive trilaterations. In this paper, we identify a class of these robots, which we call flagged manipulators, whose singularity loci admit a well-behaved decomposition, i.e., a stratification, derived from that of the flag manifold. Two remarkable properties must be highlighted. First, the decomposition has the same topology for all members in the class, irrespective of the metric details of each particular robot instance. Thus, we provide explicitly all the singular strata and their connectivity, which apply to all flagged manipulators without any tailoring. Second, the strata can be easily characterized geometrically, because it is possible to assign local coordinates to each stratum (in the configuration space of the manipulator) that correspond to uncoupled rotations and/or translations in the workspace.
\end{abstract}

Index Terms-Flag manifold, kinematics singularities, parallel manipulators, trilateration.

\section{INTRODUCTION}

$\mathbf{T}$ HE Stewart-Gough platform triggered research on parallel manipulators, and it has remained one of the most widely studied, together with its numerous specializations (designs in which the extremities of the legs merge into multiple spherical pairs). Such specializations have at least 16 forward kinematic solutions, but for two cases, in which the number of solutions goes down to 8 . These interesting cases, the 3-2-1 and 3/2 parallel manipulators, were first identified by Hunt and Primrose [1], after whom they are generically labeled.

A related nice property of Hunt-Primrose manipulators is that their forward kinematics can be derived through a sequence of three trilateration operations, i.e., by solving three tetrahedra whose vertices lie in either the platform or the base of the manipulator [2], [3]. In accordance with this, the singularity loci of these manipulators consist of those configurations for which the volume of at least one tetrahedron vanishes [4]. By analyzing the

Manuscript received May 9, 2005. This paper was recommended for publication by Associate Editor J. Angeles and Editor F. Park upon evaluation of the reviewers' comments. The work of F. Thomas and C. Torras was supported in part by the Spanish Committee for Science and Technology (CICYT) under Project DPI2004-07358, and in part by the Catalan Research Commission through the Robotics and Control Group. The work of M. Alberich-Carramiñana was supported in part under DGYCIT BFM2002-01240 and DGYCIT BFM2003-06001, and in part by the Programa Ramón y Cajal of the Ministerio de Ciencia y Tecnología.

C. Torras and F. Thomas are with the Institut de Robòtica i Informàtica Industrial (CSIC-UPC), 08028 Barcelona, Spain (e-mail: torras@ @iri.upc.edu; fthomas@iri.upc.edu).

M. Alberich-Carramiñana is with the Institut de Robòtica i Informàtica Industrial (CSIC-UPC), 08028 Barcelona, Spain. She is also with the Department of Applied Mathematics I, Technical University of Catalonia (UPC), 08028 Barcelona, Spain (e-mail: maria.alberich@upc.edu).

Digital Object Identifier 10.1109/TRO.2005.858861 pure condition (a construct used to assess the rigidity of structures), Downing et al. [5] arrived at the same conclusion. For the 3-2-1 manipulator, they showed that the pure condition is the product of three determinants, each involving the coordinates of four endpoints of the manipulator legs. Singularities occur when the pure condition vanishes, i.e., when any of the determinants becomes zero, and each determinant can be interpreted as the volume of one tetrahedron.

This provides an analytic characterization of singularities, but it does not say much about their nature and the topology of their singularity loci in the configuration space of the platform with respect to the base. While the nature of singularities for some specializations of the Stewart-Gough platform has been successfully addressed by using Grassmann line geometry [6], [7], their topological study remains as an important open problem [8]. Indeed, a complete characterization of the arrangement of singularity hypersurfaces in the configuration space would permit identifying the different nonsingular regions separated by singularities, the restriction on maneuverability occurring in each singular region, as well as the adjacencies between all nonsingular and singular regions. This would be most useful for manipulator design, including the use of redundant actuators [9] or joint coupling [10] to eliminate certain singularities, and also to plan trajectories away from singularities or, at least, from "dangerous" ones, whose unconstrained motion points toward the singularity manifold itself, thus preventing the manipulator from leaving it [11]. Furthermore, knowing the location of singularity hypersurfaces would permit crossing them in a controlled way and, therefore, operating in much larger workspaces.

In this paper, we fully characterize the topology of the singularity loci for a class of Hunt-Primrose manipulators. This class consists of particular cases of the 3/2 and the 3-2-1 manipulators satisfying the following constraints: 1) all leg endpoints in the base are coplanar; 2) all leg endpoints in the platform are coplanar; and 3) setting aside the three legs converging to the same platform attachment, the endpoints of the remaining three legs are aligned. Note that constraint 2 ) is always satisfied for the 3-2-1 manipulator, since it has a triangular platform, while constraint 3 ) is always satisfied for the $3 / 2$ manipulator, since it has only five base attachments.

The 12 manipulators in this class, obtained from the $3 / 2$ and 3-2-1 manipulators by making some of their legs share the same attachments to the base or the platform, have singularity loci with exactly the same structure, which is an unfolding of a submanifold of the flag manifold. This manifold admits a well-behaved topological decomposition, namely, a stratification, which is a partition into a finite set of submanifolds (called strata), such that the boundary of a stratum is the union 

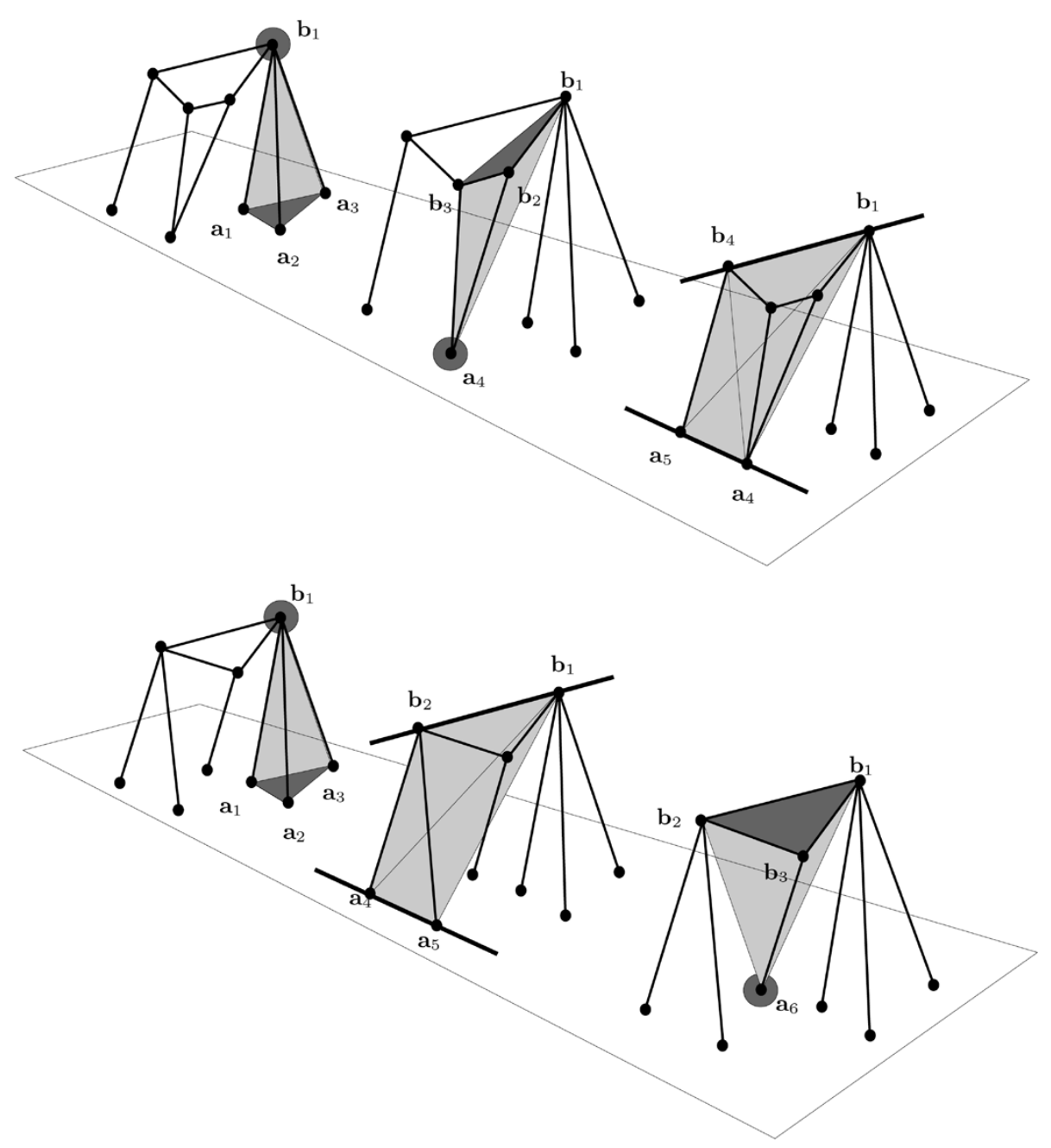

Fig. 1. Sequence of three trilaterations required to solve the forward kinematics of the 3/2 (top) and 3-2-1 (bottom) parallel manipulators.

of entire strata of lower dimensionalities. Therefore, through the unfolding, we obtain a complete characterization of the singularity submanifolds from dimension 5 down, as well as their adjacencies.

The paper is structured as follows. In the next section, Hunt-Primrose manipulators are described and their singularity loci are analytically derived. After introducing the notions of flag and stratification, Section III details the stratification of the flag manifold and the algorithmic procedure to generate it. The next two sections are devoted to the stratifications of the singularity locus of the $3 / 2$ manipulator with planar platform and base, and the 3-2-1 manipulator with planar base and the endpoints of the 2-1 legs aligned. These two parallel manipulators, together with their 10 specializations, will be referred to as flagged manipulators, and places Hunt-Primrose manipulators and their subset of flagged ones in the context of the 35 specializations of the Stewart-Gough platform defined by Faugère and Lazard [12]. Finally, some conclusions and prospects for future work are sketched in Section VII.

\section{Parallel Manipulators Solvable By Trilateration}

The spatial parallel manipulators can abstractly be described as two bodies, base and platform, so that the location of one with respect to the other is obtained from the lengths of six segments (or legs), $\overline{\mathbf{a}_{1} \mathbf{b}_{1}}, \ldots, \overline{\mathbf{a}_{6} \mathbf{b}_{6}}$, where the points $\mathbf{a}_{i}$ and $\mathbf{b}_{i}$ (or endpoints) with $i=1, \ldots, 6$ are located on the base and the platform, respectively. In what follows, we use the same notation for an endpoint and its vector of homogeneous coordinates in a given global reference frame, i.e., we will say that the homogeneous coordinates of point $\mathbf{a}$ is given by the vector $\mathbf{a}=\left(a_{x}, a_{y}, a_{z}, 1\right)$.

To simplify the direct kinematics problem, that is, that of finding the location of the platform with respect to the base, some of the endpoints either on the base or on the platform can be made coincident. Among all these possible specializations, those whose direct kinematics can be solved by a sequence of three trilaterations are called trilaterable manipulators. They were first identified by Hunt and Primrose as the 3/2 and 3-2-1 manipulators [1]. Next, we detail the required sequence of trilaterations for both manipulators, and how the analytic expression for their singularities straightforwardly follows from this sequence.

\section{A. The 3/2 Parallel Manipulator}

In a $3 / 2$ manipulator, some of the endpoints coincide in the way shown in Fig. 1 (top). Given the lengths of the segments $\overline{a_{1} b_{1}}, \overline{a_{2} b_{1}}$, and $\overline{a_{3} b_{1}}$, there are two possible mirror locations 
for $\mathbf{b}_{1}$ with respect to the plane defined by $\mathbf{a}_{1}, \mathbf{a}_{2}$, and $\mathbf{a}_{3}$. Once one of these two solutions for $\mathbf{b}_{1}$ is chosen, $\mathbf{a}_{4}, \mathbf{b}_{1}, \mathbf{b}_{2}$, and $\mathbf{b}_{3}$ define another tetrahedron with known edge lengths. Again, there are two possible mirror locations for $\mathbf{a}_{4}$, in this case with respect to the plane defined by $\mathbf{b}_{1}, \mathbf{b}_{2}$, and $\mathbf{b}_{3}$. Finally, after choosing one of the two solutions, $\mathbf{a}_{4}, \mathbf{a}_{5}, \mathbf{b}_{1}$, and $\mathbf{b}_{4}$ define another tetrahedron with known edge lengths. In this case, there are two mirror locations for $\mathbf{b}_{4}$ with respect to the plane defined by $\mathbf{a}_{4}, \mathbf{a}_{5}$, and $\mathbf{b}_{1}$. We conclude that if, and only if (iff), the points in the sets $\left\{\mathbf{a}_{1}, \mathbf{a}_{2}, \mathbf{a}_{3}, \mathbf{b}_{1}\right\},\left\{\mathbf{a}_{4}, \mathbf{b}_{1}, \mathbf{b}_{2}, \mathbf{b}_{3}\right\}$, and $\left\{\mathbf{a}_{4}, \mathbf{a}_{5}, \mathbf{b}_{1}, \mathbf{b}_{4}\right\}$ form nondegenerate tetrahedra, there are eight possible configurations for the moving platform compatible with a given set of leg lengths. Otherwise, the platform is in a singularity. In algebraic terms, iff one of the following three condition holds:

$$
\left|\begin{array}{l}
\mathbf{a}_{1} \\
\mathbf{a}_{2} \\
\mathbf{a}_{3} \\
\mathbf{b}_{1}
\end{array}\right|=0, \quad\left|\begin{array}{l}
\mathbf{a}_{4} \\
\mathbf{b}_{1} \\
\mathbf{b}_{2} \\
\mathbf{b}_{3}
\end{array}\right|=0, \quad\left|\begin{array}{l}
\mathbf{a}_{4} \\
\mathbf{a}_{5} \\
\mathbf{b}_{1} \\
\mathbf{b}_{4}
\end{array}\right|=0
$$

the platform is in a singularity. Alternatively, this condition can be expressed in a single algebraic condition as

$$
\left|\begin{array}{l}
\mathbf{a}_{1} \\
\mathbf{a}_{2} \\
\mathbf{a}_{3} \\
\mathbf{b}_{1}
\end{array}\right| \cdot\left|\begin{array}{l}
\mathbf{a}_{4} \\
\mathbf{b}_{1} \\
\mathbf{b}_{2} \\
\mathbf{b}_{3}
\end{array}\right| \cdot\left|\begin{array}{l}
\mathbf{a}_{4} \\
\mathbf{a}_{5} \\
\mathbf{b}_{1} \\
\mathbf{b}_{4}
\end{array}\right|=0
$$

The set of configurations of the platform that satisfy (2) is referred to as its singular locus. This set can also be obtained by computing the zero locus of the determinant of the analytic Jacobian associated with the parallel platform [5], but the present derivation is clearly much simpler.

Now, the problem is to express the conditions in (1) as functions of the configuration of the platform. The solution to this problem curiously comes from the developments in motion planning for polyhedra. Indeed, the three equations in (1) can be seen as the implicit equations of hypersurfaces in $\mathbb{R}^{3} \times \mathrm{SO}(3)$ associated with each of the three basic contacts between polyhedra [13], namely, the face-vertex, vertex-face, and edge-edge contacts, respectively. The algebraic expressions for these hypersurfaces, known as $\mathrm{C}$-surfaces, were first given in [14]. They are rather long (particularly the one corresponding to the third determinant), thus requiring a computer algebra system for their manipulation.

\section{B. The 3-2-1 Parallel Manipulator}

A similar analysis to that just given for the $3 / 2$ parallel manipulator, can be carried out for the 3-2-1 manipulator [Fig. 1, (bottom)]. In this case, it can be checked that iff the points in the sets $\left\{\mathbf{a}_{1}, \mathbf{a}_{2}, \mathbf{a}_{3}, \mathbf{b}_{1}\right\},\left\{\mathbf{a}_{4}, \mathbf{a}_{5}, \mathbf{b}_{1}, \mathbf{b}_{2}\right\}$, and $\left\{\mathbf{a}_{6}, \mathbf{b}_{1}, \mathbf{b}_{2}, \mathbf{b}_{3}\right\}$ form nondegenerate tetrahedra, there are eight possible configurations for the moving platform compatible with a given set of leg lengths. Otherwise, the platform is in a singularity. In algebraic terms, we have that iff

$$
\left|\begin{array}{l}
\mathbf{a}_{1} \\
\mathbf{a}_{2} \\
\mathbf{a}_{3} \\
\mathbf{b}_{1}
\end{array}\right| \cdot\left|\begin{array}{l}
\mathbf{a}_{4} \\
\mathbf{a}_{5} \\
\mathbf{b}_{1} \\
\mathbf{b}_{2}
\end{array}\right| \cdot\left|\begin{array}{l}
\mathbf{a}_{6} \\
\mathbf{b}_{1} \\
\mathbf{b}_{2} \\
\mathbf{b}_{3}
\end{array}\right|=0
$$

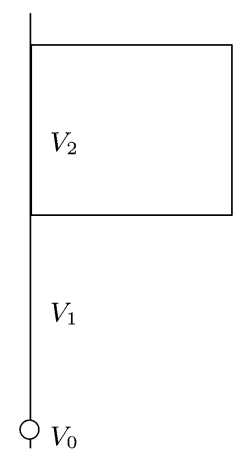

Fig. 2. A flag consists of a point $V_{0}$, a line $V_{1}$, and a plane $V_{2}$, such that $V_{0} \subset V_{1} \subset V_{2}$.

holds, the platform is in a singularity. Again, each of the three determinants in (3) equal to zero can be seen as the implicit equation of a $\mathrm{C}$-surface in $\mathbb{R}^{3} \times \mathrm{SO}(3)$ associated with each of the three basic contacts between polyhedra. In this case, with the face-vertex, edge-edge, and vertex-face contacts, respectively.

\section{The Topology of Singularities}

Each of the three C-surfaces, a variety of dimension five, involved in the description of the singularities of either the $3 / 2$ or the 3-2-1 manipulator, divides $\mathbb{R}^{3} \times \mathrm{SO}(3)$ into two half-spaces. The three $\mathrm{C}$-surfaces lead to a partition of this space into regions with congruent signs for the three determinants. The stratification of the set defined by (2) [respectively, (3)] provides a topological description of this partition.

Next we show that for the class of Hunt-Primrose manipulators satisfying the constraints mentioned in the Introduction (i.e., with planar platform and base, and three leg endpoints aligned), such a stratification can be obtained by relying on the stratification of the flag manifold without applying any algebraic manipulation, just by combinatorial considerations.

\section{Flag MANifold AND its Relation TO PARALLEL MANIPULATORS}

In this section, the notions of flag and stratification are introduced, and a result concerning the stratification of the flag manifold is reviewed, which will be subsequently used to characterize the structure of the singularity locus of the $3 / 2$ manipulator.

Definition 1 (Flag): A flag in projective space $\mathbb{P}^{3}$ is a sequence $V_{0} \subset V_{1} \subset V_{2} \subset \mathbb{P}^{3}$ of projective subspaces such that $\operatorname{dim}\left(V_{i}\right)=i$.

Let $\Pi_{\infty}$ be the plane at infinity in $\mathbb{P}^{3}$. Then the Euclidean space $\mathbb{R}^{3}$ can be viewed as a subspace of $\mathbb{P}^{3}$ via $\mathbb{R}^{3} \cong \mathbb{P}^{3} \backslash \Pi_{\infty}$. The flags we will be concerned with are the affine flags, that is, flags $V_{0} \subset V_{1} \subset V_{2} \subset \mathbb{P}^{3}$ satisfying $V_{0} \notin \Pi_{\infty}$. The name "flag" comes from the fact that it can be schematically drawn as such (see Fig. 2). Note that in drawing this figure and in all subsequent geometric interpretations, we make a slight abuse of language by identifying subspaces of dimensions 0,1 , and 2 in projective space $\mathbb{P}^{3}$ not contained in $\Pi_{\infty}$, with points, lines, and planes in Euclidean space $\mathbb{R}^{3}$. Moreover, the point, line, and plane defining a flag will referred to as "flag features." 


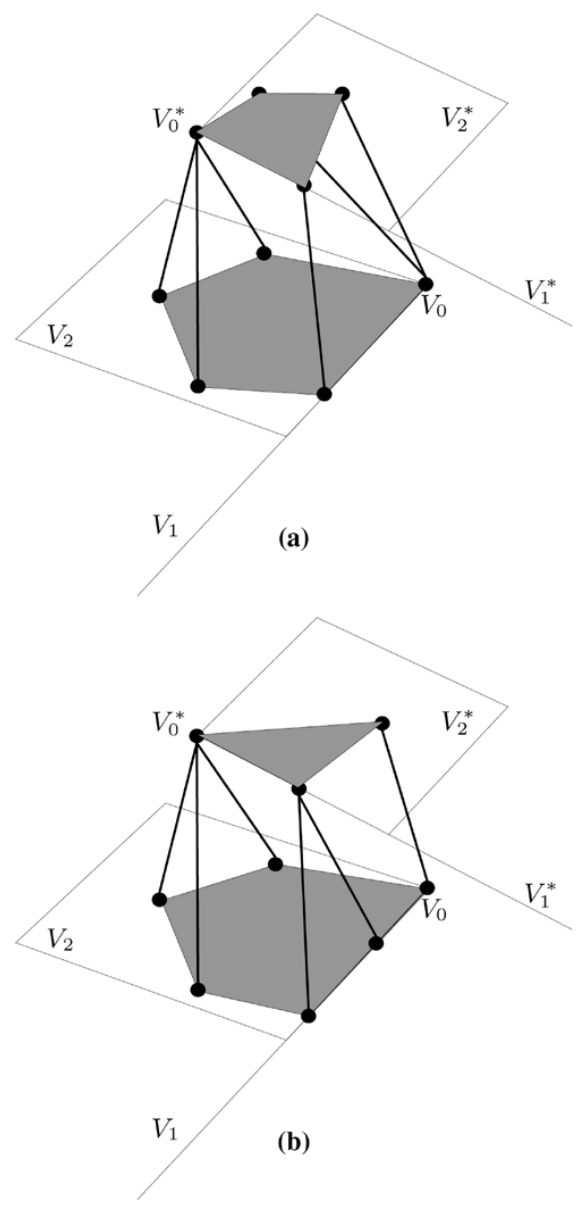

Fig. 3. Attaching a pair of flags (a) to the $3 / 2$ manipulator, and (b) to the 3-2-1 manipulator having the endpoints of the 2-1 legs aligned. Referring to the endpoint labels of Fig. 1, the flags are placed as follows: (a) $V_{0}=\mathbf{a}_{4}, V_{1}=$ $\overline{\mathbf{a}_{4} \mathbf{a}_{5}}, V_{0}^{*}=\mathbf{b}_{1}, V_{1}^{*}=\overline{\mathbf{b}_{1} \mathbf{b}_{4}}$. (b) $V_{0}=\mathbf{a}_{6}, V_{1}=\overline{\mathbf{a}_{4} \mathbf{a}_{5}}, V_{0}^{\prime}=\mathbf{b}_{1}, V_{1}^{*}=$ $\overline{\mathbf{b}_{1} \mathbf{b}_{2}}$.

For some parallel manipulators, which will hereafter be called flagged manipulators, we can attach a flag to its base and another to its platform in such a way that their singularity locus is characterized by the contact between flag features. For instance, in the case of the 3/2 manipulator with planar base and platform, the two flags will be placed as shown in Fig. 3(a). As mentioned in the preceding section, the singularity locus consists of those configurations for which any of the three trilateration operations involves a zero-volume tetrahedron. In flag terms, this occurs when either the vertex of one flag lies on the plane of the other flag or the two flag lines intersect.

Now, we are interested in decomposing the singularity locus into simple manifolds, as well as obtaining their adjacency relationships. As we will show next, the topologically distinct configurations of the manipulator correspond to distinct incidence relations between the features of their attached flags.

This relates to the space of all flag configurations. We will first introduce this space and a well-known result concerning its topological decomposition, and next apply it to the singularity locus of interest.

Definition 2 (Flag Manifold): The flag manifold $\mathcal{F l a g}(4)$ is the set of all flags in $\mathbb{P}^{3}$. Let $\mathcal{F}_{\mathcal{A}}$ denote the subset of the affine flags in $\mathcal{F l a g}(4)$.
Choose coordinates in $\mathbb{P}^{3}$ such that the embedding of $\mathbb{R}^{3}$ into $\mathbb{P}^{3}$ is given by $\left(x_{1}, x_{2}, x_{3}\right) \rightarrow\left(x_{1}, x_{2}, x_{3}, 1\right)$. The flags whose vertex has coordinates $\left(x_{1}, x_{2}, x_{3}, 0\right)$, that is, lies on $\Pi_{\infty}$, form a submanifold of $\mathcal{F} \operatorname{lag}(4)$ of codimension 1 . Hence, the set of the affine flags $\mathcal{F}_{\mathcal{A}}\left(\mathbb{P}^{3}\right)$ is an open subset of $\mathcal{F l a g}(4)$.

The notion of well-behaved topological decomposition is formalized in the following definition.

Definition 3 (Stratification): A stratification of a set $S$ is a partition $S=\cup_{i \in I} S_{i}$ such that:

1) $I$ is finite;

2) $S_{i}$ is a manifold $\forall i$;

3) if $S_{i} \cap \bar{S}_{j} \neq \emptyset$, then $S_{i} \subset \bar{S}_{j}$, where $\bar{S}_{j}$ stands for the closure of $S_{j}$.

The $S_{i}$ 's are called strata. The third item in the list is a boundary condition, which is included to guarantee that the boundary of a stratum is the union of the entire strata of lower dimensionalities.

Next, we introduce the main result on the stratification of the flag manifold. Its derivation is outlined in the Appendix and, for a more detailed exposition, the reader is referred to [15] and [16].

It is possible to stratify the 6-D $\mathcal{F} \operatorname{lag}(4)$ into strata $B^{w}$ (each $B^{w}$ isomorphic to $\mathbb{R}^{\text {length }(w)}$, see the Appendix) in such a way that every permutation $w$ in $\sum_{4}$ identifies a unique stratum

$$
\mathcal{F} \operatorname{lag}(4)=\cup_{w \in \sum_{4}} B^{w}
$$

where $\sum_{4}$ stands for the set of permutations of four elements. Furthermore, two strata of consecutive dimensions are adjacent iff there is a single transposition between their associated permutations. This provides an algorithmic procedure to generate the graph of strata for the flag manifold.

Following this procedure, we have derived the stratification shown in Fig. 4, where each row includes the strata of the same dimension, starting with dimension 0 at the bottom to dimension 6 at the top.

The notation used in this figure requires some explanation. We characterize the reference frame by means of a reference flag (which is an affine flag), and then label each stratum with the incidence relations between the flags in this stratum and the reference flag. Hence, $v, l$, and $p$ stand for the point, line, and plane conforming the reference flag, while the same letters with an asterisk stand for the same elements of the other flag; a hyphen between two elements means that one is included in the other, while a dot denotes that they meet at a single point. In all, we have characterized each stratum by means of three items: 1) a symbolic description using the notation above; 2) the matrix $\mathbf{M}$ of dimensions of the intersections between the elements of the two flags, where an empty intersection is denoted by -1 , a point intersection by 0 , etc.; and 3 ) its associated permutation. The permutation is computed as follows: add to $\mathbf{M}$ a first row $(-1,-1,-1)$ and a last row $(0,1,2)$ to obtain an extended $5 \times 3$ matrix, and let $\mathbf{m}_{i}, 1 \leq i \leq 3$ denote its three columns; if the permutation is $w=(w(1), w(2), w(3), w(4))$, then $w(i), 1 \leq i \leq 3$ is the new position of the element of $\mathbf{m}_{i}$ at which there is a jump in dimensionality, and $w(4)$ completes the permutation with the remaining digit (see the Appendix for a detailed example). 


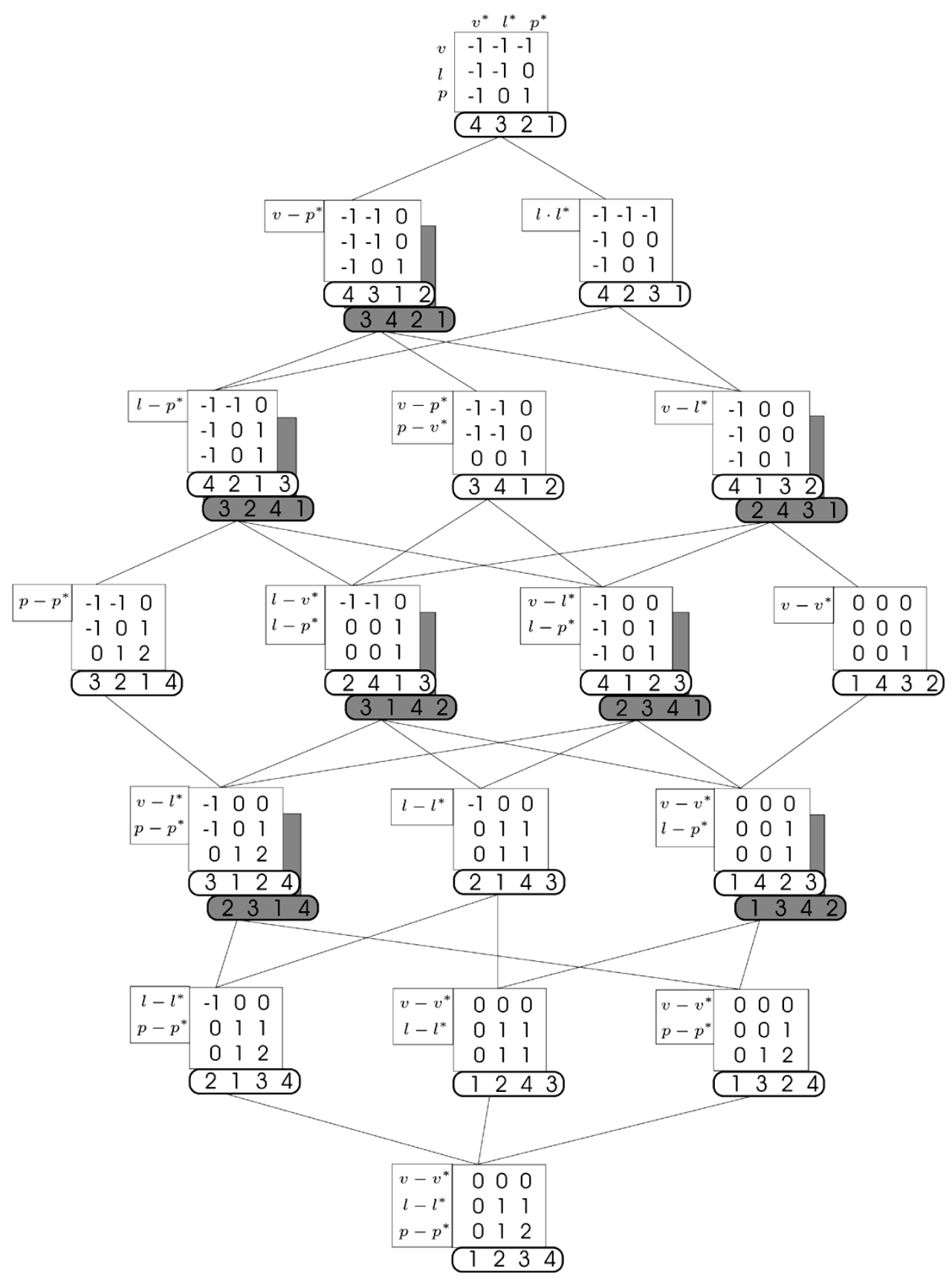

Fig. 4. Graph showing the stratification of $\mathcal{F} \operatorname{lag}(4)$.

Actually, one should proceed from 3) to 1). One starts with the graph of permutations, where arcs join permutations that differ in a single transposition. From each permutation, the corresponding matrix is derived by introducing the jumps in dimensionality, as specified above. And, finally, the symbolic description follows straightforwardly from the matrix, by interpreting its entries as incidences between the elements of the two flags, assigned to rows and columns, respectively.

Note that strata having symmetric matrices correspond to incidence relations that remain invariant when the roles of the two flags are interchanged. Conversely, nonsymmetric matrices result in a pair of different strata under such interchange, whose matrices are the transposes of one another. Due to space limitations, only one of every such pair of strata is fully shown, the other being represented only by its associated permutation, which is shown shaded.

The stratification of $\mathcal{F} \operatorname{lag}(4)$ induces a stratification of $\mathcal{F}_{\mathcal{A}}\left(\mathbb{P}^{3}\right)$

$$
\mathcal{F}_{\mathcal{A}}\left(\mathbb{P}^{3}\right)=\cup_{w \in \sum_{4}}\left(B^{w} \cap \mathcal{F}_{\mathcal{A}}\left(\mathbb{P}^{3}\right)\right) .
$$

Since the reference flag is an affine flag, none of the above intersections is empty. However, it might happen that some cell $B^{w}$ would split off into two connected components [17]: for instance, for $w=(4,2,3,1)$, the affine flags of $B^{w}$ are the disjoint union of the two cells $\left\{\left(v^{*}, l^{*}, p^{*}\right) \in B^{w}: v^{*}=\right.$ $\left.\left(x_{1}, x_{2}, 1, x_{4}\right), x_{1}, x_{2}, x_{4} \in \mathbb{R}, x_{4}>0\right\}$ and $\left\{\left(v^{*}, l^{*}, p^{*}\right) \in\right.$ $\left.B^{w}: v^{*}=\left(x_{1}, x_{2}, 1, x_{4}\right), x_{1}, x_{2}, x_{4} \in \mathbb{R}, x_{4}<0\right\}$. Indeed, $B^{w} \cap \mathcal{F}_{\mathcal{A}}$ is a unique cell iff the permutation $w$ starts with 


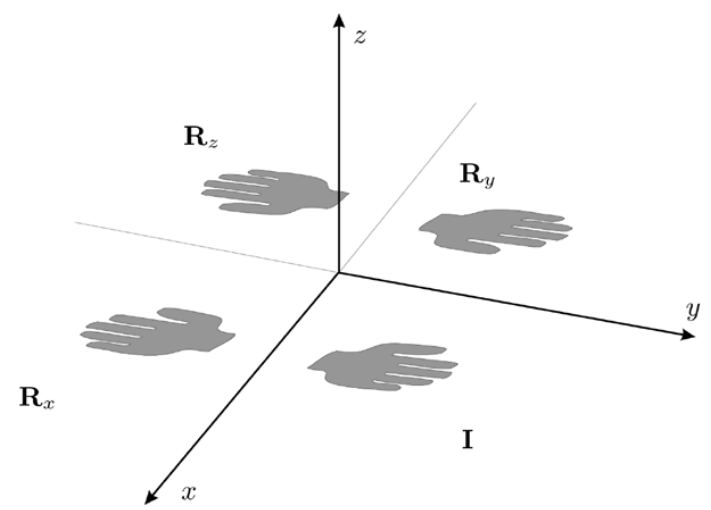

Fig. 5. Group of Euclidean transformations that leaves a flag invariant. The flag consists of the origin, the $x$ axis, and the $x y$ plane.

$w(1)=1$, that is, the stratum label contains the incidence relation $v-v^{*}$.

\section{STRATIFYING THE SingUlaRITY LOCUS OF THE 3/2 MANIPULATOR}

Now we return to the setting above of a $3 / 2$ manipulator holding two flags, one attached to the base and another to the platform as in Fig. 3(a), and take up again the question of how many relative configurations they can adopt with distinct incidence relations between their flag features. Note that considered within the flag, features are unoriented (lines and planes), while considered as platform features (edges and faces), they are oriented.

Given a flag $\mathcal{V}^{*}=\left(V_{0}^{*}, V_{1}^{*}, V_{2}^{*}\right)$ attached to the $3 / 2$ manipulator platform as in Fig. 3(a), we can define a reference frame having $V_{0}^{*}$ as origin, $V_{1}^{*}$ as the $x$ axis, and $V_{2}^{*}$ as the $x y$ plane. This flag remains invariant when a rotation of $\pi$ radians about any of the three coordinate axes is applied (see Fig. 5). Formally, the group of Euclidean transformations leaving the flag invariant is $\mathcal{H}_{\mathcal{V}^{*}}=\left\{\mathbf{I}, \mathbf{R}_{x}, \mathbf{R}_{y}, \mathbf{R}_{z}\right\}$, where $\mathbf{I}$ is the identity transformation, and $\mathbf{R}_{k}$ stands for a rotation of $\pi$ radians about the $k$-axis. Let us mention that $\mathcal{H}_{\mathcal{V}}$ is one of the representations of the well-known abelian group $C_{2} \times C_{2}$ [18], since $\mathbf{R}_{x} \mathbf{R}_{y}=\mathbf{R}_{y} \mathbf{R}_{x}=\mathbf{R}_{z}, \mathbf{R}_{x} \mathbf{R}_{z}=\mathbf{R}_{z} \mathbf{R}_{x}=\mathbf{R}_{y}$, and $\mathbf{R}_{y} \mathbf{R}_{z}=\mathbf{R}_{z} \mathbf{R}_{y}=\mathbf{R}_{x}$.

We can attach a reference frame to the base of the $3 / 2$ manipulator from the reference flag $\mathcal{V}=\left(V_{0}, V_{1}, V_{2}\right)$ in a similar way as above. Then, a manipulator configuration is described as $\mathbf{q} \in \mathbb{R}^{3} \times \mathrm{SO}(3)$, relating the platform frame to the base frame. Given one such manipulator configuration $\mathbf{q}$, with its associated flag configuration, we can characterize the set of four manipulator configurations yielding this same flag configuration, as follows:

$$
\left\{\mathbf{q} \mathbf{T} \mid \mathbf{T} \in \mathcal{H}_{\mathcal{V}_{\mathbf{q}}^{*}}\right\}
$$

where $\mathcal{V}_{\mathbf{q}}^{*}$ is the flag associated with the platform in configuration $\mathbf{q}$. This gives a four-fold covering map $\pi: \mathbb{R}^{3} \times \mathrm{SO}(3) \rightarrow$ $\mathcal{F}_{\mathcal{A}} \cdot{ }^{1}$ Therefore, with each relative configuration of two flags,

\footnotetext{
${ }^{1}$ This map corresponds to the restriction of what in algebraic geometry is known as the four-fold covering map between the partially oriented flag manifold $G(1,1 \mid 1,1)$ in $\mathbb{P}^{3}$ and $\mathcal{F} \operatorname{lag}(4)$ [19].
}

we can associate four relative configurations of the platform and base (Fig. 6). It is worth mentioning that these four configurations leading to the same flag arrangement do not have anything to do with the eight direct kinematic solutions of the manipulator. To visualize this, consider the four manipulator configurations corresponding to a given flag arrangement; obviously, each such configuration will have a different set of leg lengths (refer again to Fig. 6). Conversely, the eight manipulator configurations with the same leg lengths will have associated different flag arrangements (Fig. 7).

The covering morphism $\pi$ induces a stratification of $\mathbb{R}^{3} \times$ $\mathrm{SO}(3)$, and also of the singularity locus $X$ of the $3 / 2$ manipulator, from the stratification of $\mathcal{F}_{\mathcal{A}}\left(\mathbb{P}^{3}\right)$ obtained in the preceding section. Namely, (6) provides a procedure to unfold the graph in Fig. 4 into a stratification of $X$. Going into details, there are three cells in $\mathcal{F l a g}(4)$ of 5-D singularities, which correspond to the cases in which point $\mathbf{b}_{1}$ of the platform lies on the base plane, point $\mathbf{a}_{4}$ of the base lies on the platform plane, and segments $\overline{\mathbf{a}_{4} \mathbf{a}_{5}}$ and $\overline{\mathbf{b}_{1} \mathbf{b}_{4}}$ lie on a plane [see Fig. 1, (top)]. Restricted to $\mathcal{F}_{\mathcal{A}}\left(\mathbb{P}^{3}\right)$, they split off into six cells. Due to the four degrees of $\pi$, this leads in $\mathbb{R}^{3} \times \mathrm{SO}(3)$ to 24 5-D strata separated by $5 \times 2 \times 4$ 4-D strata, whose adjacencies follow directly from the permutation structure of the flag manifold. In the same way, we can derive the $5 \times 2 \times 4+1 \times 43$-D strata, the $3 \times 2 \times 4+2 \times 42$-D strata, the $1 \times 2 \times 4+2 \times 41$-D strata, the $1 \times 40$-D strata, and their adjacencies. Note that for the reasons mentioned in the preceding section, all strata $B^{w}$ of $\mathcal{F} \operatorname{lag}(4)$ whose label contains the incidence relation $v-v^{*}$ (or equivalently, their defining permutation $w$ begins with one) are entirely composed of affine flags, hence, they remain the same when restricted to $\mathcal{F}_{\mathcal{A}}$, and thus, $\pi^{-1}\left(B^{w}\right)$ has lower cardinality.

In all, there are 160 singular strata separating eight disjoint 6-D strata, corresponding to the eight connected components of the nonsingular manipulator configurations, which (by connectness arguments) must equal the eight regions with congruent signs for the three determinants introduced in Section II-C.

An interesting point about this stratification of the singularity locus is that each stratum encompasses a subset of the three pure translations and three pure rotations, along and about the coordinate axes defined by the flags. Thus, by appropriately choosing local coordinates, each stratum can be made to correspond to uncoupled translations and/or rotations in the workspace. Moreover, in following down the stratification adjacencies (see Fig. 4), one degree of freedom (DOF) at a time is lost. For example, the 4-D strata of type $v-l^{*}$ consist of the three rotations plus the translation along the line $l^{*}$, and, in moving to its frontier 3-D strata of type $v-v^{*}$, the translational DOF is lost (Fig. 8).

\section{Stratifying the Singularity Locus OF THE 3-2-1 MANIPULATOR}

In the case of the 3-2-1 manipulator, it is possible to associate a flag to its platform, but not to its base, even if it is planar. This is because the base point used in the third trilateration does not necessarily lie in the line used for the second trilateration [see Fig. 1, (bottom)]. Therefore, it is not a flagged manipulator. 

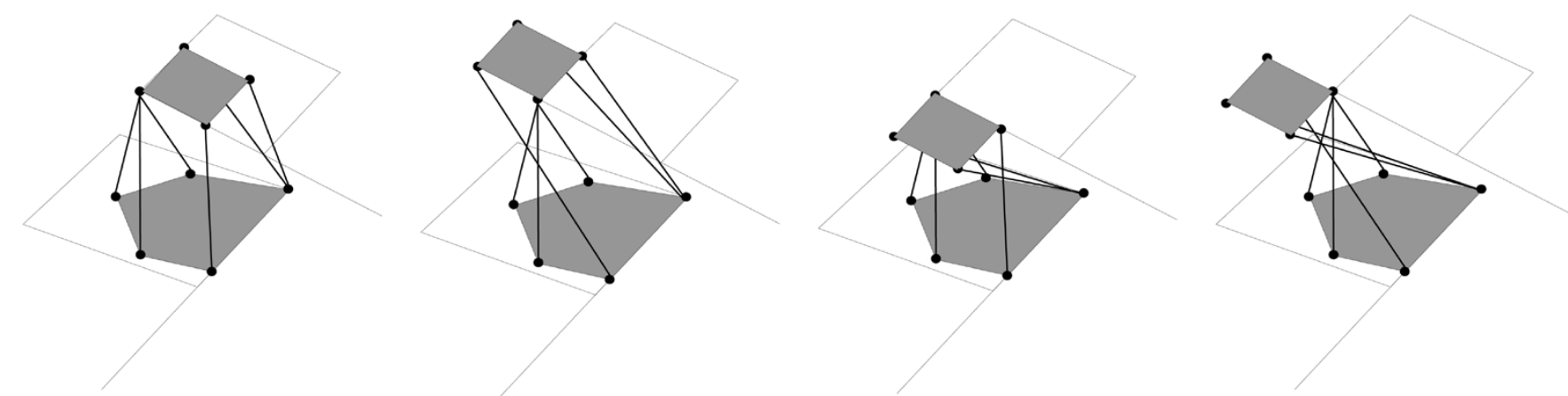

Fig. 6. Four different configurations of the platform with respect to the base of a flagged manipulator lead to the same configuration for the attached flags.
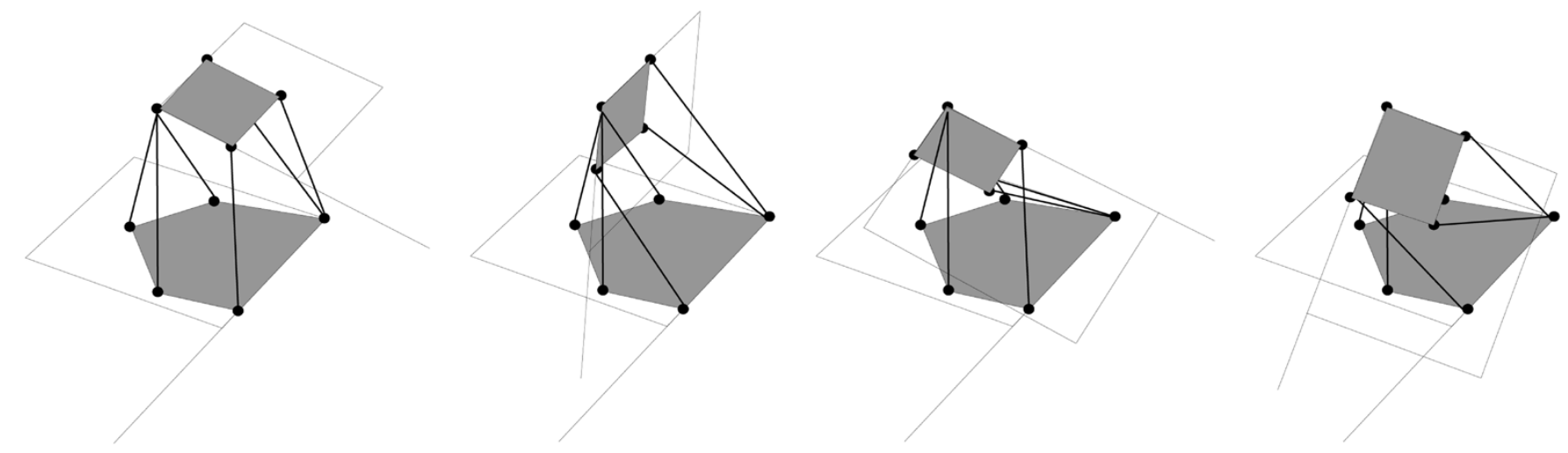

Fig. 7. Given a set of leg lengths, a flagged manipulator can have up to eight configurations compatible with them, which, in general, lead to different configurations for the attached flags. For the sake of simplicity, the four mirror configurations with respect to the base plane are not shown.

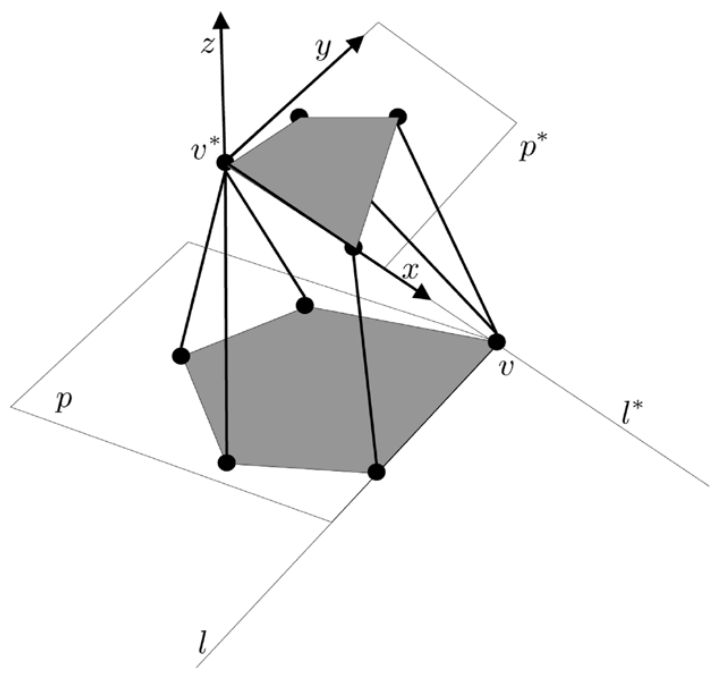

Fig. 8. 4-D stratum of type $v-l^{*}$ of the singularity locus of this flagged manipulator can be fully described geometrically by a spherical rotation centered at $v$ and a translation along $l^{*}$. In moving to its frontier 3-D stratum of type $v-v^{*}$, the translational DOF is lost. This characterization is the same for all flagged manipulators.

Of course, as for all trilaterable manipulators, a stratification of its singularity locus can be obtained by intersecting the C-surfaces corresponding to a zero-volume tetrahedron in any of the three trilateration operations. However, the resulting strata do not correspond to uncoupled DOFs.
The special 3-2-1 manipulator having the endpoints of the 2-1 legs aligned is a flagged manipulator, and its singularity locus has exactly the same structure as that of the $3 / 2$ manipulator.

\section{Placing Trilaterable and Flagged MANIPULATORS IN CONTEXT}

It is reasonable to ask ourselves for the importance of trilaterable and flagged manipulators in the universe of all parallel manipulators. Beyond qualitative considerations about constructive simplicity and frequency of use, we have approached this question from the viewpoint of the combinatorial classes of parallel manipulators established by Faugère and Lazard [12].

Starting from the general 6-6 manipulator with six linear actuators connected by spherical joints to the platform and to the base, they listed 60 classes differing in their associated graph of legs. Quite understandably, the 6-6 manipulator was denoted

the 3-2-1 manipulator

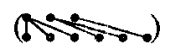

and the $3 / 2$ manipulator 


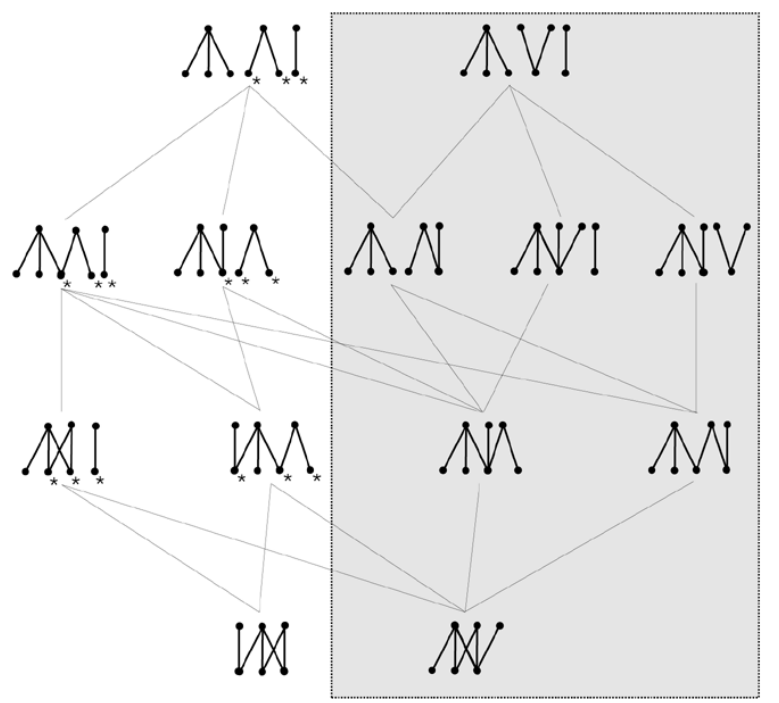

Fig. 9. Trilaterable manipulators: the 3-2-1 manipulator (top left), the $3 / 2$ manipulator (top right), and all their specializations. When both the platform and the base are planar, the manipulators in the grey area are directly flagged, while the remaining ones, except the bottom left, are flagged, provided that three of their endpoints are aligned (here marked with an asterisk).

When identifying two classes that are deduced one from the other by exchanging the platform and the base, the number of classes reduces to 35 . We will not reproduce the whole list here, but just detail the trilaterable and flagged manipulators.

Fig. 9 shows a representation of the 13 trilaterable manipulators and their specialization relations.

Let us recall that in the case of the $3 / 2$ family (those in the grey area), the trilateration sequence involves two vertex-plane tetrahedra followed by an edge-edge tetrahedron (refer to Fig. 1), while for the 3-2-1 family, the sequence starts with a vertexplane tetrahedron, proceeds with a edge-edge one, and ends up again with a vertex-plane tetrahedron. Of course, in the case of manipulators belonging to the two families, either sequence works.

Now, let us restrain our analysis to manipulators having a planar platform and a planar base. Then the seven members of the $3 / 2$ family are flagged manipulators. Note that three of them come purely from this family, namely the $3 / 2$ itself

\section{(A...}

and its two specializations

\section{(AN D) \\ (A.)}

The remaining four flagged manipulators, while still belonging to the $3 / 2$ family, are also specializations of the 3-2-1 manipulator

$$
\left(N_{0}\right),(D+N),(1 \times)
$$

Five additional manipulators belonging to the 3-2-1 family (those not in the grey area in Fig. 9, except the bottom left one) are flagged, provided that the endpoints of their 2-1 legs are aligned. These are the 3-2-1 manipulator itself

\section{$(1 \cdots)$}

and four of its specializations

$$
\text { (AN.), (NN), (ND), (LN) }
$$

The remaining one

\section{(BD)}

does not admit the alignment of their endpoints without falling into a degenerate configuration.

To summarize, among the 35 classes of parallel manipulators, 13 are trilaterable. By restraining the classification to manipulators satisfying the three conditions stated in the Introduction, namely: 1) all leg endpoints in the base are coplanar; 2) all leg endpoints in the platform are coplanar; and 3) setting aside the three legs converging to the same platform attachment, the endpoints of the remaining three legs are aligned, it turns out that 12 of the trilaterable classes are now flagged ones.

It is worth mentioning that some of the specializations above have received particular attention from the kinematics community, their singularity loci having been analytically derived. For example, in the case of the

\section{(N)}

manipulator, Collins and McCarthy [20] concluded that its singularity locus is a cubic surface that factors into three planes, which can now be viewed as a direct consequence of its being a trilaterable manipulator. Moreover, since it satisfies the three conditions stated above, it is also a flagged manipulator, and its singularity locus is known to admit the stratification presented in this paper.

\section{CONCLUSION}

The singularity loci of parallel manipulators have been the subject of analytic, numeric, and geometric studies. Obtaining the singularities analytically requires finding the roots of the Jacobian determinant, which is rather difficult in many cases. $\mathrm{Nu}$ merical procedures have been developed and applied to overcome this difficulty. Both approaches characterize the singularity loci, but do not provide information on either the nature of the singularities or their location in the configuration space of the manipulator. By applying Grassmann line geometry, the Jacobian is interpreted as a matrix of leg line vectors, and singularities are viewed as line configurations with rank lower than six, which can be classified according to their dimensionality reduction and geometric nature. Thus, line geometry answers one of the above needs, but still leaves open the question of how 
singular configurations are distributed throughout the configuration space.

In this paper, we have addressed this latter question in the case of the 3/2 and 3-2-1 parallel manipulators by applying a combinatorial topology result, namely, the stratification of the flag manifold.

For these manipulators, the Jacobian determinant can be factored into three determinants involving the coordinates of four leg endpoints each. The loci of these three determinants are hypersurfaces in configuration space (C-surfaces), which can be interpreted as the three well-known basic contacts between two polyhedra, namely, vertex-face, face-vertex, and edge-edge contacts, the former two corresponding to endpoint-base and platform-endpoint incidences in the present context.

The intersections of the above hypersurfaces lead to singularity regions of lower dimensionalities. In this paper, we have shown that for the $3 / 2$ manipulator with planar platform and base, and the 3-2-1 manipulator with planar base and the 2-1 leg endpoints aligned, as well as for their 10 specializations, the three singularity hypersurfaces admit a well-behaved topological partition, which is a four-fold covering of the stratification of a submanifold of the flag manifold.

Thus, we have obtained a complete description of the singularity locus for a whole class of in-parallel manipulators, consisting of 160 strata together with all their topological adjacencies. The strata are submanifolds of dimensions from 5 down to 0 , which can be parameterized by means of uncoupled rotations and/or translations related to some axes of the manipulator. It is remarkable that the obtained stratification has the same topology for all members in the class, irrespective of the metric details of each particular manipulator instance.

It should also be mentioned that this stratification is purely kinematic, in the sense that it does not take into account interferences that may occur between the platform, the base and the legs, or bounds in the actuator's range of operation. Further work is needed to trim the above general stratification to adapt it to the metrics and bounds of a particular physical platform.

As a practical application, we will undertake this trimming for the 3-2-1 pose measuring device [4], which will allow us to assess the interest of the proposed singularity characterization for trajectory planning and control.

\section{APPENDIX}

Definition 4 (Grassmannian): A Grassmannian in $\mathbb{P}^{d}$ is a manifold

$G(k, d)=\left\{V \subset \mathbb{P}^{d} \mid V\right.$ is a linear variety and $\left.\operatorname{dim}(V)=k\right\}$.

The flag manifold $\mathcal{F} l a g(d+1)$ can thus be expressed in the following way:

$$
\begin{aligned}
\mathcal{F} \operatorname{lag} & (d+1) \\
= & \left\{\left(V_{0}, V_{1}, \ldots V_{d-1}\right)\right. \\
& \in G(0, d) \times G(1, d) \times \cdots \times G(d-1, d) \mid V_{0} \\
& \left.\subset V_{1} \subset \cdots \subset V_{d-1}\right\} .
\end{aligned}
$$

Definition 5 (Subspace Signature): Given a fixed reference flag $\left(V_{0}^{r}, V_{1}^{r}, \ldots, V_{d-1}^{r}\right)$ in $\mathbb{P}^{d}$, the signature $s\left(V^{*}\right)$ of $V^{*} \in$ $G(k, d)$ is a sequence $\left(1 \leq s_{0}<s_{1}<\cdots<s_{k} \leq d+1\right)$, where the $s_{i} \in \mathbb{N}$ are the "jump points" for $V^{*}$, i.e., $V^{*} \cap V_{s_{i}-2}^{r} \neq$ $V^{*} \cap V_{s_{i}-1}^{r}$ (Note that $V_{-1}^{r}=\emptyset$ and $V_{d}^{r}=\mathbb{P}^{d}$ ).

If $F^{*}=\left(V_{0}^{*}, V_{1}^{*}, \ldots, V_{d-1}^{*}\right) \in \mathcal{F} \operatorname{lag}(d+1)$, we get a triangular array of signatures

$$
\begin{aligned}
s\left(V_{0}^{*}\right) & =s_{00} \\
s\left(V_{1}^{*}\right) & =s_{10}<s_{11} \\
\vdots & \\
s\left(V_{d-1}^{*}\right) & =s_{(d-1) 0}<s_{(d-1) 1}<\cdots<s_{(d-1)(d-1)} .
\end{aligned}
$$

Lemma 1: ([15, Ch. 3, Lemma 4.2]) If $V \subseteq V^{\prime}$, with $V$ and $V^{\prime}$ subspaces of $\mathbb{P}^{d}$, then $s(V) \subseteq s\left(V^{\prime}\right)$.

Hence, as one goes down the rows in (7), exactly one new number is added at each stage, so one gets a sequence $\left\{s_{00}, s_{1 i_{1}}, \ldots, s_{(d-1) i_{(d-1)}}\right\}$ of distinct numbers between 1 and $d+1$. If we add the remaining number at the end, we get a permutation $w \in \sum_{(d+1)}$.

Definition 6 (Flag Signature): The signature $\hat{s}$ of the flag $F^{*}=\left(V_{0}^{*}, V_{1}^{*}, \ldots, V_{d-1}^{*}\right)$ above is $\hat{s}\left(F^{*}\right)=w$.

Example 1: Keep the notations of Fig. 4. Let $F^{*}=$ $\left(V_{0}^{*}, V_{1}^{*}, V_{2}^{*}\right)=\left(v^{*}, l^{*}, p^{*}\right) \in \mathcal{F} \operatorname{lag}(4)$ be a flag whose incidence relations with the reference flag $\left(V_{0}^{r}, V_{1}^{r}, V_{2}^{r}\right)=(v, l, p)$ are only $l \cdot l^{*}$. The extended matrix (extended to $V_{-1}^{r}=\emptyset$ and $V_{3}^{r}=\mathbb{P}^{3}$ ) of dimensions of the intersections between flag features is, in this case

$$
\left(\begin{array}{ccc}
-1 & -1 & -1 \\
-1 & -1 & -1 \\
-1 & 0 & 0 \\
-1 & 0 & 1 \\
0 & 1 & 21
\end{array}\right) .
$$

We have $s\left(v^{*}\right)=4, s\left(l^{*}\right)=2<4, s\left(p^{*}\right)=2<3<$ $4 ;\left\{s_{00}, s_{10}, s_{21}\right\}=\{4,2,3\}$; and completing the permutation with the remaining digit $\hat{s}\left(F^{*}\right)=w=(4,2,3,1)$.

Definition 7 (Bruhat Cell): A Bruhat cell is $B^{w}=\{F \in$ $\mathcal{F} \operatorname{lag}(d+1) \mid \hat{s}(F)=w\}$.

Theorem 1: ([15, Ch. 3, Th. 4.3]) $\mathcal{F l a g}(d+1)$ is the disjoint union of all Bruhat cells $B^{w}$ with $w \in \sum_{(d+1)}$.

Definition 8 (Length): The length of a permutation $w \in \sum_{(d+1)}$ is

$$
\text { length }(w)=|\{(i<j) \mid w(i)>w(j)\}| \text {. }
$$

Proposition 1: ([15, Ch. 3, Prop. 4.7]) $\operatorname{dim}\left(B^{w}\right)=$ length $(w)$. Moreover, $B^{w}$ is isomorphic to $\mathbb{R}^{\text {length }(w)}$.

Definition 9 (Cover): If $w, w^{\prime} \in \sum_{(d+1)}$, we say that $w^{\prime}$ covers $w$, and we denote it by $w \rightarrow w^{\prime}$, if there exists a transposition $t$ such that $w^{\prime}=t w$ and length $(t w)=$ length $(w)+1$.

Definition 10 (Bruhat Order): The Bruhat order $<$ is the transitive closure of $\rightarrow$.

Definition 11 (Closure of a Bruhat Cell): The closure of a Bruhat cell $B^{w}$ is

$$
\bar{B}^{w}=\{F \in \mathcal{F} \operatorname{lag}(d+1) \mid \hat{s}(F) \leq w\}=\bigcup_{w^{\prime} \leq w} B^{w^{\prime}}
$$

which is known as the Schubert variety.

Theorem 1, together with the property just mentioned, are the conditions required for

$$
\mathcal{F} \operatorname{lag}(d+1)=\bigcup_{w \in \sum_{(d+1)}} B^{w}
$$


to constitute a stratification of $\mathcal{F} \operatorname{lag}(d+1)$.

Summarizing, each stratum can be identified by its associated permutation, and the adjacencies between strata of consecutive dimensionalities can be derived directly from the Bruhat order.

\section{ACKNOWLEDGMENT}

The authors thank F. Guillén for drawing their attention to the flag manifold.

\section{REFERENCES}

[1] K. H. Hunt and E. J. F. Primrose, "Assembly configurations of some in-parallel actuated manipulators," Mech. Mach. Theory, vol. 28, no. 1, pp. 31-42, 1993.

[2] H. Bruyninckx, "Forward kinematics for Hunt-Primrose parallel manipulators," Mech. Mach. Theory, vol. 34, no. 4, pp. 657-664, 1999.

[3] S.-K. Song and D.-S. Kwon, "A tetrahedron approach for a unique closed-form solution of the forward kinematics of six-DOF parallel mechanisms with multiconnected joints," J. Robot. Syst., vol. 19, no. 6, pp. 269-281, 2002.

[4] F. Thomas, E. Ottaviano, L. Ros, and M. Ceccarelli, "Performance analysis of a 3-2-1 pose estimation device," IEEE Trans. Robot., vol. 21, no. 3, pp. 288-297, Jun. 2005.

[5] D. M. Downing, A. E. Samuel, and K. H. Hunt, "Identification of the special configurations of the octahedral manipulator using the pure condition,” Int. J. Robot. Res., vol. 21, no. 2, pp. 147-160, 2002.

[6] J. P. Merlet, "Singular configurations of parallel manipulators and Grassmann geometry," Int. J. Robot. Res., vol. 8, no. 5, pp. 45-56, 1992.

[7] A. Wolf and M. Shohan, "Investigation of parallel manipulators using linear complex approximation," Trans. ASME, J. Mech. Des., vol. 125, pp. 564-572, 2003.

[8] B. Dasgupta and T. S. Mruthyunjaya, "The Stewart platform manipulator: A review," Mech. Mach. Theory, vol. 35, no. 1, pp. 15-40, 2000.

[9] G. Liu, Y. Lou, and Z. Li, "Singularities of parallel manipulators: A geometric treatment," IEEE Trans. Robot. Autom., vol. 19, no. 4, pp. 579-594, Aug. 2003.

[10] I.-M. Chen, J. Angeles, Theingi, and C. Li, "Shaping singularity loci of parallel manipulators using joint-coupling," in Proc. 7th Int. Conf. Autom. Technol., Chia-Yi, Taiwan, R.O.C., 2003, pp. 515-520.

[11] V. K. Chan and I. Ebert-Uphoff, "Investigation of the deficiencies of parallel manipulators in singular configurations through the Jacobian nullspace," in Proc. IEEE Int. Conf. Robot. Autom., Seoul, Korea, 2001, pp. 1313-1320.

[12] J. C. Faugère and D. Lazard, "Combinatorial classes of parallel manipulators," Mech. Mach. Theory, vol. 30, no. 6, pp. 765-776, 1995.

[13] F. Thomas and C. Torras, "A projectively invariant intersection test for polyhedra," Vis. Comput., vol. 18, no. 7, pp. 405-414, 2002.

[14] B. R. Donald, "A search algorithm for motion planning with six degrees of freedom," Artif. Intell., vol. 31, pp. 295-353, 1987.

[15] H. Hiller, Geometry of Coxeter Grups. London, U.K.: Pitman, 1982, Research Notes in Mathematics.

[16] D. Monk, "The geometry of flag manifolds," Proc. London Math. Soc., vol. 9, no. 9, pp. 253-286, 1959 .

[17] M. Alberich-Carramiñana, V. González, and C. Torras. (2005) The topology of singularities of flagged parallel manipulators, IRI Tech. Rep. [Online]. Available: http://www-iri.upc.es/people/torras

[18] G. Arfken, Mathematical Methods for Physicists, 3rd ed. Orlando, FL: Academic, 1985, pp. 244-245.
[19] P. Sankaran and P. Zvengrowski, "Stable parallelizability of partially oriented flag manifolds II," Can. J. Math., vol. 49, no. 6, pp. 1323-1339, 1997.

[20] C. L. Collins and J. M. McCarthy, "The singularity loci of two triangular parallel manipulators," in Proc. IEEE Int. Conf. Adv. Robot., 1997, pp. 473-479.

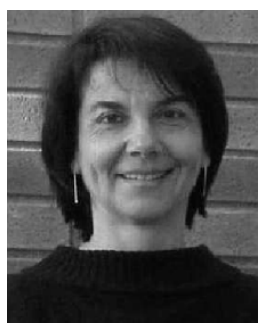

Carme Torras received the M.Sc. degrees in mathematics and computer science from the University of Barcelona, Barcelona, Spain, and the University of Massachusetts, Amherst, respectively, and the Ph.D. degree in computer science from the Technical University of Catalonia, Catalonia, Spain.

She is currently a Research Professor with the Spanish Scientific Research Council (CSIC), Barcelona, Spain. She has published four books and more than 100 papers in the areas of robot kinematics, geometric reasoning, computer vision, and neurocomputing. She has been local project leader of several European projects, such as "Planning RObot Motion" (PROMotion), "Robot Control based on Neural Network Systems" (CONNY), "Self-organization and Analogical Modeling using Subsymbolic Computing" (SUBSYM), and "Behavioral Learning: Sensing and Acting" (B-LEARN).

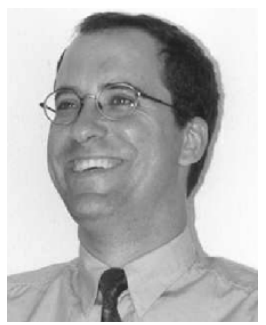

Federico Thomas received the B.Sc. degree in telecommunications engineering in 1984, and the $\mathrm{Ph} . \mathrm{D}$. degree (with honors) in computer science in 1988, both from the Technical University of Catalonia, Catalonia, Spain.

Since March 1990, he has been a Research Scientist with the Institut de Robòtica i Informàtica Industrial, Spanish High Council for Scientific Research, Barcelona, Spain. His research interests are in geometry and kinematics, with applications to robotics, computer graphics, and computer vision. He has been project leader of several national projects financed by the Spanish Committee for Science and Technology (CICYT), and by local companies such as ENHER, a power generation company now part of ENDESA.

In 1991, he won a NATO postdoctoral scholarship at the University of Massachusetts, Amherst, with the late Prof. R. Popplestone. He is an Associate Editor of the IEEE TRANSACTIONS ON ROBOTICS.

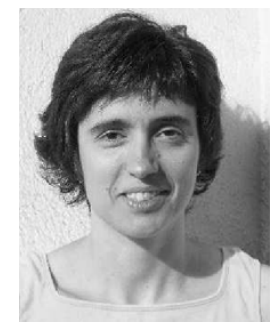

Maria Alberich-Carramiñana received the M.Sc. and Ph.D. degrees in mathematics, both from the Universitat de Barcelona, Barcelona, Spain, in 1994 and 2000 , respectively.

In 2001, she joined the Technical University of Catalonia (UPC), Barcelona, Spain, where she is currently a Researcher, holding a "Ramón y Cajal" position. Her research areas are singularity theory, algebraic geometry, and their applications to robotics and computer vision. She is the author of the book Geometry of the Plane Cremona Maps (New York: Springer, 2002, ser. Lecture Notes in Mathematics). 